\title{
A Robust Method to Integrate End-to-End Mission Architecture Optimization Tools
}

\author{
Rafael Lugo \\ Analytical Mechanics Associates, Inc. \\ 21 Enterprise Parkway Suite 300 \\ Hampton, VA 23666 \\ 757-864-7147 \\ rafael.a.lugo@nasa.gov
}

\author{
Daniel Litton \\ NASA Langley Research Center \\ Mail Stop 489 \\ Hampton, VA 23681 \\ 757-864-4117 \\ daniel.k.litton@nasa.gov
}

\author{
Min Qu \\ Analytical Mechanics Associates, Inc. \\ 21 Enterprise Parkway Suite 300 \\ Hampton, VA 23666 \\ 757-864-9737 \\ min.qu-1@nasa.gov
}

\author{
Jeremy Shidner \\ Analytical Mechanics Associates, Inc. \\ 21 Enterprise Parkway Suite 300 \\ Hampton, VA 23666 \\ 757-864-4516 \\ jeremy.d.shidner@nasa.gov
}

\author{
Richard Powell \\ Analytical Mechanics Associates, Inc. \\ 21 Enterprise Parkway Suite 300 \\ Hampton, VA 23666 \\ 757-864-4506 \\ richard.w.powell@nasa.gov
}

\begin{abstract}
End-to-end mission simulations include multiple phases of flight. For example, an end-to-end Mars mission simulation may include launch from Earth, interplanetary transit to Mars and entry, descent and landing. Each phase of flight is optimized to meet specified constraints and often depend on and impact subsequent phases. The design and optimization tools and methodologies used to combine different aspects of end-to-end framework and their impact on mission planning are presented. This work focuses on a robust implementation of a Multidisciplinary Design Analysis and Optimization (MDAO) method that offers the flexibility to quickly adapt to changing mission design requirements. Different simulations tailored to the liftoff, ascent, and atmospheric entry phases of a trajectory are integrated and optimized in the MDAO program Isight, which provides the user a graphical interface to link simulation inputs and outputs. This approach provides many advantages to mission planners, as it is easily adapted to different mission scenarios and can improve the understanding of the integrated system performance within a particular mission configuration. A Mars direct entry mission using the Space Launch System (SLS) is presented as a generic end-to-end case study. For the given launch period, the SLS launch performance is traded for improved orbit geometry alignment, resulting in an optimized a net payload that is comparable to that in the SLS Mission Planner's Guide.
\end{abstract}

\section{TABLE OF CONTENTS}

1. INTRODUCTION 1

2. CASE STUdy: MARS DiRect EnTRY .................... 2

3. TRAJECTORY SIMULATION.................................... 4

4. OPTIMIZATION FRAMEWORK .................................... 5

5. RESULTS................................................................ 6

6. SUMMARY AND CONCLUSIONS ........................... 7

ACKNOWLEDGEMENTS .......................................... 7

REFERENCES.................................................... 7

BIOGRAPHY ........................................................... 8

\section{INTRODUCTION}

In early conceptual phases of mission architecture design, mission planners often adjust objectives and constraints that require re-optimization of the spacecraft trajectory to establish accurate performance measures. The updated results inform the mission planners to modify the design and, if necessary, re-optimize. The cycle continues until a satisfactory mission architecture is achieved. Examples of conceptual end-to-end mission architecture scenarios include determining how the payload capability of a specific launch vehicle varies with (1) launch opportunity, (2) arrival destination (e.g. Mars, Venus, or Europa), or (3) location of the interplanetary injection burn (performed at perigee of an elliptical orbit versus a circular orbit). Often, these problems require simulating the spacecraft trajectory across the entire mission, or "end-to-end."

This paper presents the design and optimization tools and methodologies used to combine different aspects of end-toend analysis and their impact on mission planning. A typical end-to-end analysis utilizes different types of tools to simulate the various phases of a mission (e.g. liftoff, orbit, touchdown, etc.). Models of varying fidelity, such as those for vehicle aerodynamics and engine performance, may also be implemented in the tools.

Several methods exist for integrating the design and optimization tools and models in a cohesive environment. Some tools may provide interfaces that allow data to be input directly from a different simulation. Other methods fall under the class of Multidisciplinary Design Analysis and Optimization (MDAO), which combine the various tools and models in a global simulation and provide optimization techniques that operate globally on all of the tools. This permits the user to specify design objectives and constraints that the MDAO program achieves by dynamically adjusting model and simulation inputs based on the desired optimization technique. 
The present work focuses on a robust implementation of the MDAO methodology that offers the flexibility to quickly adapt to changing mission design requirements. A Mars direct entry mission architecture using the Space Launch System (SLS) as a launch vehicle will be presented as an end-to-end case study. High-fidelity simulations are used to model the various phases of the trajectory, including liftoff, ascent, and on-orbit operations. The optimization framework and part of the simulation are adapted from a previous end-to-end analysis conducted for Exploration Mission 1 (EM-1) [1], which further emphasizes the inherent flexibility of the process.

\section{CASe Study: Mars Direct EnTRY}

A Mars direct entry mission architecture using the SLS launch vehicle is selected to demonstrate the capabilities and flexibility of the MDAO framework and supporting analytical processes. Though not based on any scheduled or planned flight, it provides a useful example of a human precursor-class mission.

\section{Space Launch System}

The selected Mars direct entry mission architecture assumes a late October/early November 2026 launch window. SLS is NASA's heavy-lift launch vehicle solution to sending large payloads and crews to the Moon, Mars, and beyond. The nominal Block 1 crew configuration, shown in Figure 1, uses existing Space Shuttle RS-25 engines and modified solid rocket boosters (SRBs) on the Core Stage. The upper stage consists of the Interim Cryogenic Propulsion Stage (ICPS), Orion Multi-Purpose Crew Vehicle (MPCV), and Launch Abort System (LAS).

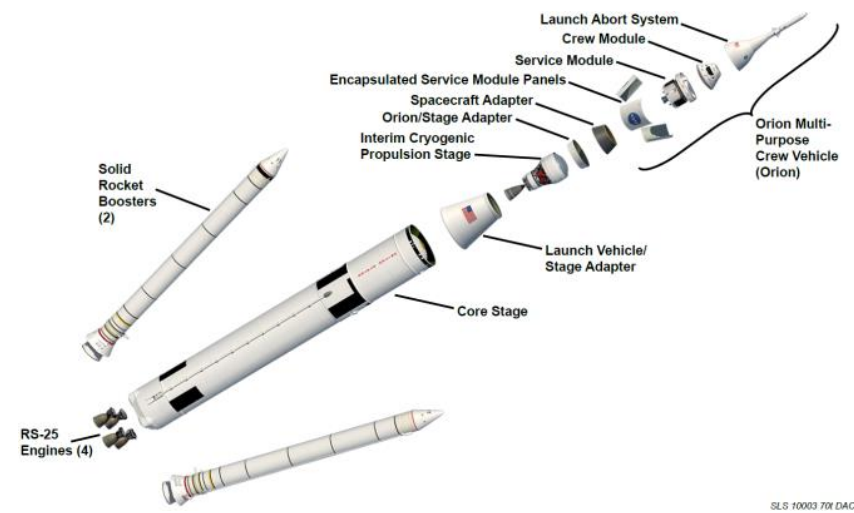

Figure 1. SLS Block 1 (crew) [2]

The Block 1 crew configuration is used for the present analysis because it is currently at a more advanced design stage than other, larger configurations [2]. Note, however, that although the analysis utilizes the same mass properties as the MPCV, no assumptions are made about the specific content of this payload. That is, the payload is a generic mass that shares the mass properties of MPCV. Figure 2 shows this "generic payload" in the context of the upper stage and corresponding spacecraft adapters.

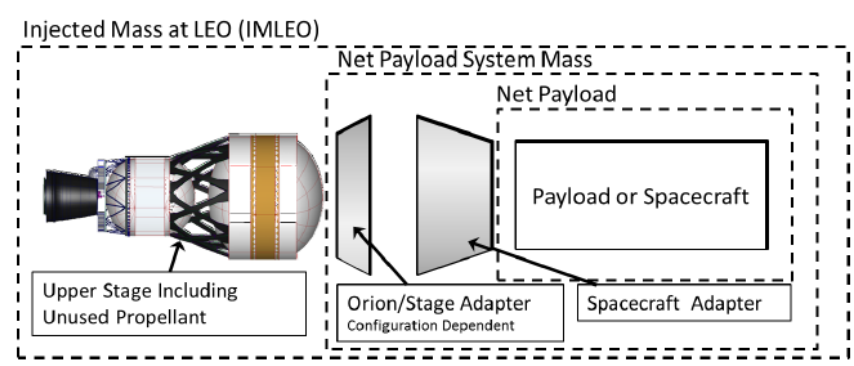

Figure 2. Mass delivery to LEO [2]

Performance characteristics of the various SLS configurations are shown in Figure 3. The SLS Block 1 (Orion + ICPS) configuration has a net payload system mass of approximately $20 \mathrm{t}$ at a $C_{3}$ of $10 \mathrm{~km}^{2} / \mathrm{s}^{2}$, which is roughly the energy required for a Mars mission at this scale. Note that this was computed assuming an Eastward launch azimuth, which permits taking full advantage of Earth's rotational velocity. Later in this paper, it will be shown that comparable performance may be achieved at launch azimuths that are not perfectly Eastward.

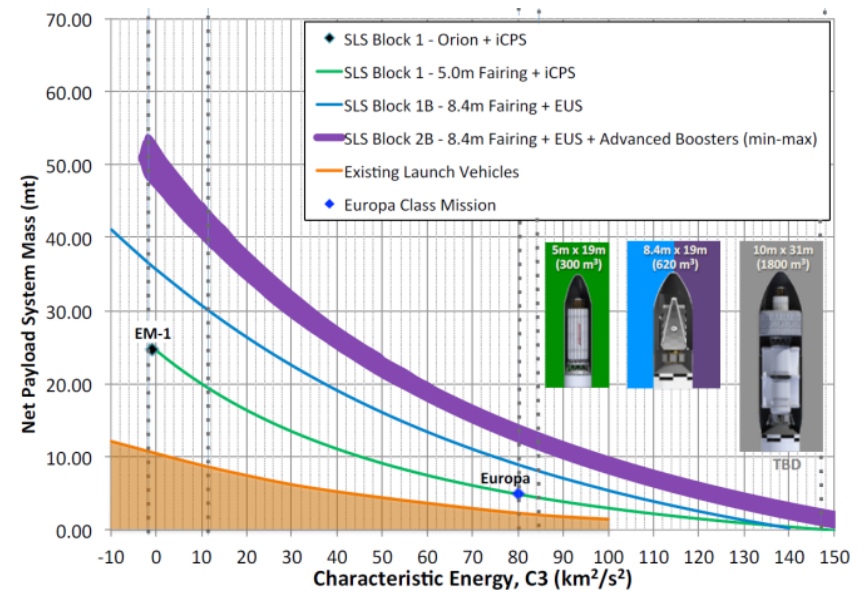

Figure 3. SLS performance characteristics [2]

\section{Concept of Operations}

The concept of operations for a Mars direct entry is shown in Figure 4. This figure is adapted from a similar diagram in the SLS Mission Planner's Guide Executive Overview [2]. Note that the figure is notional: planets and orbits are not to scale and events are not portrayed in accurate geometric locations. 


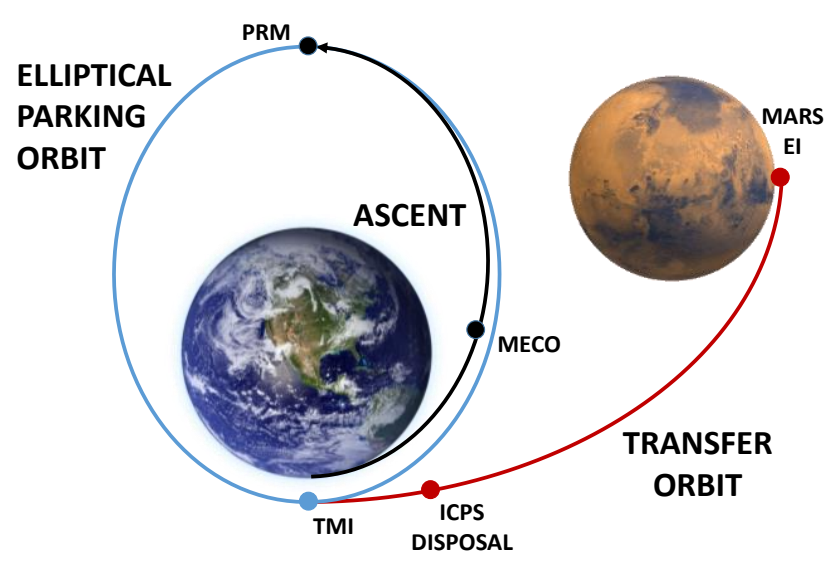

Figure 4. Mars direct entry concept of operations

The ascent portion of the trajectory begins at liftoff and goes through main engine cutoff (MECO). The SRBs, Core Stage, Service Module panels, and LAS are jettisoned during ascent. After MECO and near apogee, the ICPS performs a perigee raise maneuver (PRM) that injects the ICPS/MPCV into an elliptical parking orbit. After at least one full orbit, which provides crew and/or mission controllers time for systems checkouts, the ICPS performs the trans-Mars injection (TMI) burn near perigee that puts the MPCV into a transfer orbit to Mars. After the TMI burn, the ICPS is jettisoned. For this analysis, no further burns or maneuvers are simulated.

\section{Lambert's Problem}

From a mission planning perspective, simple analytical computations can help to define launch and arrival window parameters that warrant deeper investigation in the end-toend analysis. For an Earth/Mars mission, a Lambert solver [3] can be utilized to determine a feasible trajectory from launch and arrival time of Earth and Mars locations respectively. The solutions for the 2026 mission window analyzed here have been computed using the JPL DE418 ephemeris for the Earth-Moon barycenter and Mars state. The initial parameters considered from the Lambert solution for launch include the required $\mathrm{C} 3$ and $V_{\infty}$ declination from the Earth equator. For arrival, the parameters of interest are the entry velocity at $125 \mathrm{~km}$ altitude and the approach $V_{\infty}$ declination from the Mars equator.

The launch $C_{3}$ in Figure 5 is presented as a function of launch and arrival date, creating a figure that is commonly referred to as a "porkchop plot". The singularity near the center represents the near optimal $180^{\circ}$ Hohmann transfer between Earth and Mars while the line that runs from bottom left to top right also represents $180^{\circ}$ transfers, but the planets are too far apart in their respective orbital planes thus requiring large and inefficient inclination changes. The solutions on the bottom right of the singularity are referred to as Type I trajectories and are faster in transit than the trajectories to the upper left, or Type II trajectories.

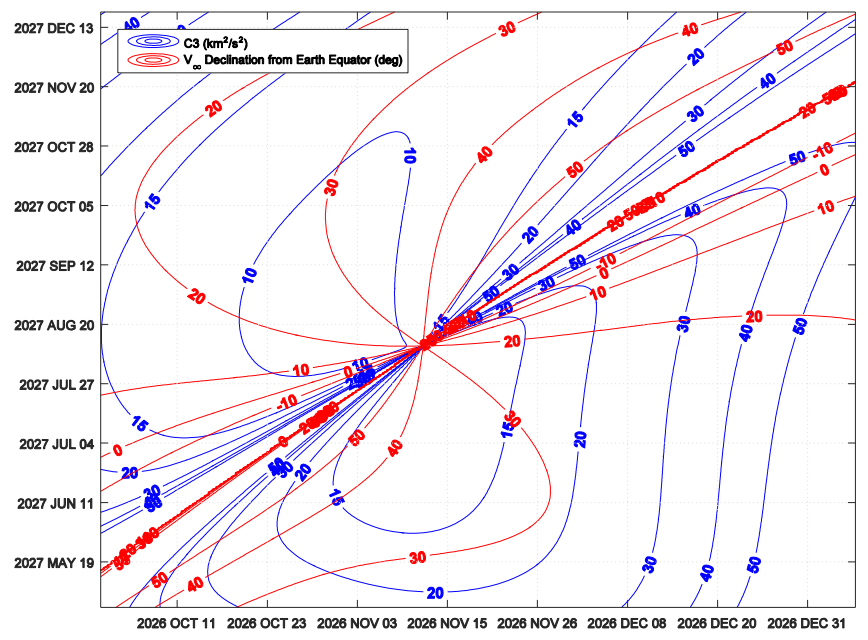

Figure 5. Lambert Solution: Characteristic Energy and Declination of Velocity Vector at Departure

For the SLS vehicle, the $C_{3}$ is directly correlated with launch capability, and is an indicator of which combination of launch and arrival days are possible for a given payload mass. For the $20 \mathrm{t}$ payload discussed in this paper, the $C_{3}$ curve of $10 \mathrm{~km}^{2} / \mathrm{s}^{2}$ is a good indicator of the mission window size. Note that the $10 \mathrm{~km}^{2} / \mathrm{s}^{2}$ class of trajectories are only available during the Type II trajectories (longer transit time). The $V_{\infty}$ declination from the Earth equator may be further used as a discriminator of the launch window because of the constraints of the higher fidelity analysis in the end-to-end tool, i.e. communications, disposal constraints or parking orbit requirements of the SLS launch. If the $C_{3}$ of $10 \mathrm{~km}^{2} / \mathrm{s}^{2}$ and $\mathrm{V}_{\infty}$ declination is limited to $10^{\circ}$, one can see the launch window quickly narrowing and providing the area of interest to focus analyses.

Arrival characteristics are also important for defining the mission window. The arrival parameters shown in Figure 6 include the $V_{\infty}$ declination of the arrival asymptote relative to the Mars equator and the entry velocity at a $125 \mathrm{~km}$ altitude above Mars. Though entry is not currently included in the optimization framework, the end-to-end tool will eventually analyze entry to understand the heating environment and communications to Earth which are often analogous to the entry velocity and declination of the asymptote. Future analysis should take into account these parameters for selecting the mission window constraints. 


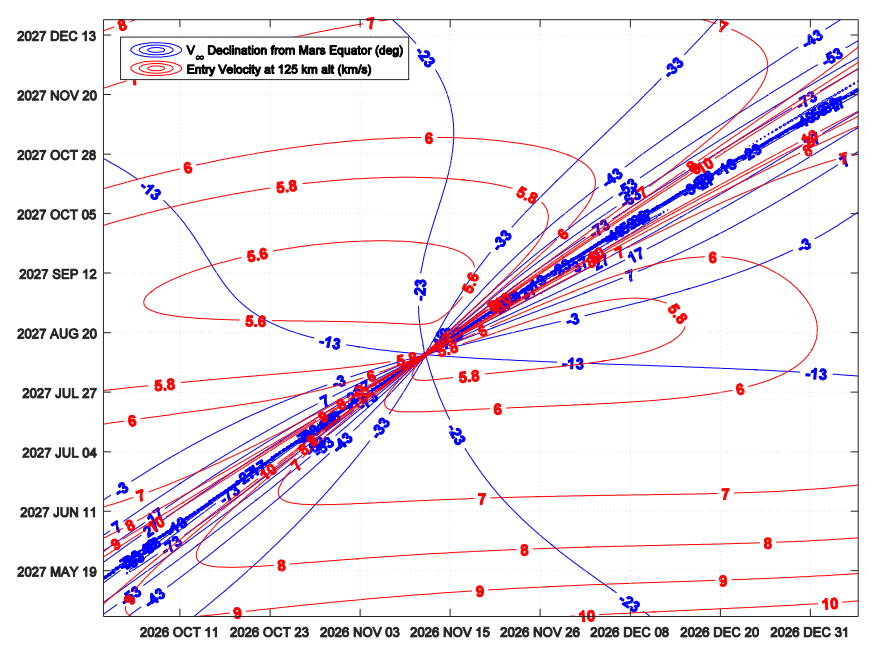

Figure 6. Lambert Solution: Declination of Velocity Vector and Entry Velocity at Arrival

\section{TRAJECTORY SIMULATION}

The Mars direct entry end-to-end mission is simulated using two high-fidelity tools within the MDAO framework, both of which are tailored to simulating specific portions of the trajectory.

\section{POST2}

Liftoff and ascent phases are simulated using a three degreeof-freedom trajectory in the Program to Optimize Simulated Trajectories II (POST2) [4]. POST2 is a generalized pointmass simulation program that has been used extensively in the design, analysis, and optimization of single and multibody vehicle trajectories in atmospheric and orbital flight.

A user-created input deck defines all initial state conditions, mass properties, and environment models. Optimization control variables and objectives may also be defined. Guidance commands and trajectory milestones such as booster jettison and main engine cutoff (MECO) are defined as "events" in the input deck. POST2 reads the input deck, integrates the equations of motion to generate the trajectory, and outputs time histories of various state variables as selected by the user. It can also output values of the various state variables at selected events.

The desire within this framework is to make the POST2 SLS Block 1 ascent simulation easily applicable to virtually any mission, regardless of final destination. This commonality is useful when analyzing other types of missions, such as a lunar flyby [1]. In fact, the ascent simulation in the present analysis is identical (that is, the POST2 input deck is unmodified) to that in [1]. The ascent simulation may be updated in the future to model other launch vehicles such as SLS Block 1B, Block 2, Atlas V, etc.

\section{Copernicus}

Earth-Moon and interplanetary transfer orbits are simulated using Copernicus, a three degree-of-freedom trajectory optimization program [5]. Copernicus may be run from either the command line or the provided graphical user interface (GUI). Figure 7 shows the Mars direct entry trajectory as seen in the Copernicus GUI (label callouts have been added for clarity). This is an Earth-centered visualization with the spacecraft trajectory in purple and the orbits of Mars and Earth in red and blue, respectively.

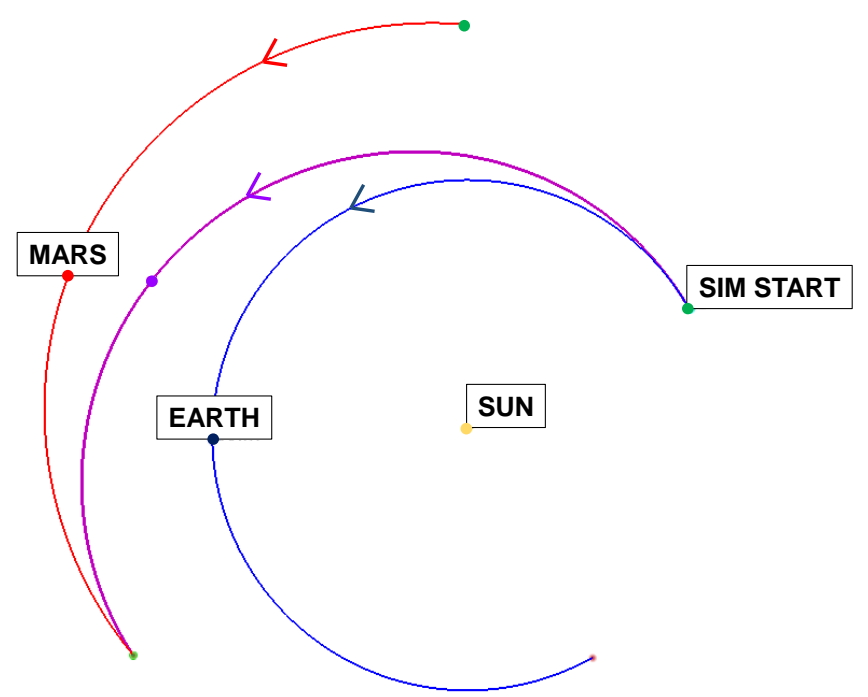

Figure 7. Mars direct entry trajectory simulated in
Copernicus

A user-created input deck defines all necessary variables to describe the trajectory. On-orbit trajectory milestones such as TMI are defined as "segments" similar in concept to events in POST2. The user may select different variables to target or optimize at these segments. For example, the user may select the optimization objective to minimize the fuel used at TMI, or maximize the final mass at Mars entry interface (EI). As is the case with POST2, Copernicus can output time histories of various state variables or at specified segments. The user-created input deck is updated with the optimized values for each segment. The fact that Copernicus uses the same file for both input and output is a notable difference from POST2, which outputs to a separate file.

Most optimization problems benefit from using a starting set of initial conditions that provide an output that is reasonably close to the optimal solution. The challenge is that the definition of "reasonable" varies between optimizers and the case being optimized. Copernicus simulation speed improves significantly when the input deck (which defines the entire trajectory) closely represents the solution. This will become relevant when analyzing trajectory solutions across a launch period. 


\section{OPTIMIZATION FRAMEWORK}

The optimization framework used in the present analysis is an extension of the EM-1 framework formulated in [1]. In keeping with the philosophy of a flexible and robust framework, other design problems may be derived from this process flow.

End-to-end MDAO integration is implemented using Isight, a commercial software package capable of combining multidisciplinary models and applications in a streamlined process flow [6]. A GUI permits click-and-drag parameter linking through the optimization flow. The Isight interface permits the user to quickly adjust initial conditions, constraints, control variables, and objective variables. Variables may be bounded or targeted. If the control variable combination leads to a solution with variables that are outside the defined bounds, that solution is poorly weighted. If the control variable combination leads to a solution with a value that does not match the target value, that solution is also poorly weighted (numerical tolerances are considered). Thus, the user may improve solution feasibility by carefully selecting which variables are to be targeted and which variables are to be bounded.

\section{Data Interchange}

End-to-end optimization requires that different simulation programs interchange data. For example, the end state of the liftoff and ascent phase of the trajectory from POST2 must be used to initialize the Copernicus simulation of the onorbit phase. Since most simulation programs are not written to directly interface with each other, data must be interchanged through output files that may be parsed or read by another program. POST2 has been modified to have the ability to output the values of specific variables at desired events in a JavaScript Object Notation (JSON) file. ${ }^{1}$ JSON is a standardized notation format that is useful for interchanging data because it is designed to facilitate parsing by different programming languages [7].

\section{Process Flow}

For a given day in the launch period, Isight selects values for the global control variables (launch azimuth and parking orbit apogee). These control variables are passed into the POST2 ascent simulation, which Isight optimizes to maximize the mass to MECO. Once the ascent trajectory is optimized, the vehicle state, orientation, and mass at MECO are written to a JSON file. Isight parses the POST2 JSON output to read the MECO state, and updates the MECO segment in the Copernicus input deck accordingly (for the first iteration, a previously converged Copernicus input deck is used). The initial guess for the duration of the TMI burn is also adjusted based on the ratio of the updated MECO mass to the MECO mass from the previous iteration, since a

\footnotetext{
${ }^{1}$ Copernicus also has the ability to output JSON files, but currently it is not a native feature and must be implemented through a user-created plugin.
}

larger mass will require a longer burn time. Copernicus then runs the updated input deck and optimizes the transfer orbit to maximize the mass to Mars entry interface. Copernicus also adjusts the MECO time to align it with the optimized transfer orbit (the POST2 solution can be shifted with the rotating Earth to launch at essentially any time). The converged output deck is saved and used to begin the next iteration.

Isight uses the resultant solution and optimization history to select a new set of control variables (launch azimuth and apogee), and begin the next iteration. Convergence is achieved when the desired solution accuracy is satisfied. Isight then increase the day of the launch period by one and the process begins again. The optimization process flow is illustrated in Figure 8. Blue boxes represent steps taken by Isight. Green boxes represent steps taken by the trajectory simulation tools POST2 and Copernicus.

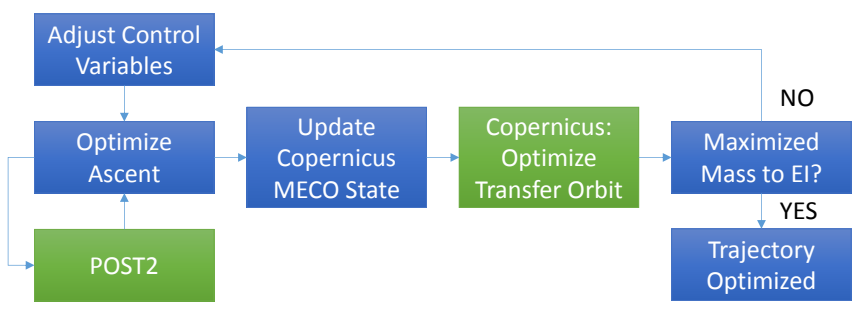

Figure 8. Optimization process flow

As the SLS program matures, the POST2 and Copernicus input decks can be modified to account for changes to the design and performance of the vehicle and to the liftoff, ascent, and transfer orbit trajectories. As long as handoff point from POST2 to Copernicus is MECO, these updates can be made with little to no modifications to the MDAO implementation in Isight.

\section{Optimization Problems}

Trajectory optimization is achieved at three steps or levels within the framework: ascent, transfer orbit, and global. Copernicus optimizers are used for on-orbit maneuvers to optimize the mass to Mars entry interface. Isight optimizers are used for ascent to optimize the mass to MECO and to drive the optimization problem on a global scale.

Ascent Optimization-The optimization objective to maximize mass to MECO is achieved by adjusting the offload mass and the various pitch control variables that affect the SLS ascent maneuvers. The offload mass is removed (or added) from the net payload; recall that the payload in this analysis is assumed to be a generic mass with mass properties of the MPCV, and therefore large variations in offload mass are deemed acceptable. Given the inputs of launch azimuth and parking orbit apogee, Isight iterates on the ascent trajectory by running POST2 many times until convergence is achieved. Optimization of the ascent portion of the trajectory uses the Large Scale Generalized Reduced Gradient (LSGRG) Optimizer in Isight [8]. 
Transfer Orbit Optimization-The optimization objective to maximize mass at Mars entry interface is achieved by adjusting several control variables, including those related to the location and duration of the perigee raise maneuver and TMI burn. Copernicus moves the true anomalies of these maneuvers so that their start location provides burns with the highest energy, but also satisfies the constraints imposed by the geometry of the required Mars transfer orbit. Optimization of the transfer orbit uses the Sparse Nonlinear Optimizer (SNOPT) in Copernicus [9].

Global Optimization-The optimization objective to maximize mass at Mars entry interface is achieved by adjusting the control variables of launch azimuth and apogee at MECO, which is passed into POST2. Adjusting these two variables permits the optimizer to find a TMI burn that occurs at or near the parking orbit perigee, which is the optimal location to burn in terms of required change in velocity. The TMI burn does not necessarily occur at perigee, however, because the Mars transfer orbit geometry may dictate a different burn location. Thus, Isight ties together the ascent and transfer orbit portions of the trajectory to find an optimal solution on the global scale. Optimization at the global level uses the Mixed Integer Sequential Quadratic Programming (MISQP) optimizer in Isight [10].

Table 1 summarizes the optimizers, objectives, controls, and constraints for each level of optimization within the framework.

Table 1. Optimization objectives, controls, and constraints

\begin{tabular}{c|c|c|c}
\hline \hline & Ascent & Transfer Orbit & Global \\
\hline Optimizer & LSGRG & SNOPT & MISQP \\
\hline \multirow{2}{*}{ Objective } & $\begin{array}{c}\text { Maximize } \\
\text { MECO mass }\end{array}$ & $\begin{array}{c}\text { Maximize Mars } \\
\text { EI mass }\end{array}$ & $\begin{array}{c}\text { Maximize Mars } \\
\text { EI mass }\end{array}$ \\
\hline \multirow{4}{*}{ Controls } & $\bullet$ Liftoff mass & $\bullet$ Perigee raise \\
& $\begin{array}{c}\bullet \text { Ljustment } \\
\text { match control } \\
\text { Constraints }\end{array}$ & $\begin{array}{c}\bullet \text { TMI maneuver } \\
\text { azimuth }\end{array}$ & $\bullet$ Apogee \\
& $\begin{array}{c}\bullet \text { SRB \& Core } \\
\text { Stage fuel use }\end{array}$ & $\begin{array}{c}\bullet \text { Entry interface } \\
\text { variables (e.g., } \\
\text { altitude, flight }\end{array}$ & $\bullet$ TMI fuel use \\
& $\bullet$ Max. dynamic & path angle) & \\
& pressure & & \\
\hline \hline
\end{tabular}

\section{RESUltS}

Results from the Mars direct entry optimization problem are shown in Figure 9 and Figure 10. For this analysis, a launch period from 23 October 2026 to 12 November 2026 was investigated. A pre-converged Copernicus input deck for the 3 November launch date was used to begin the optimization process.
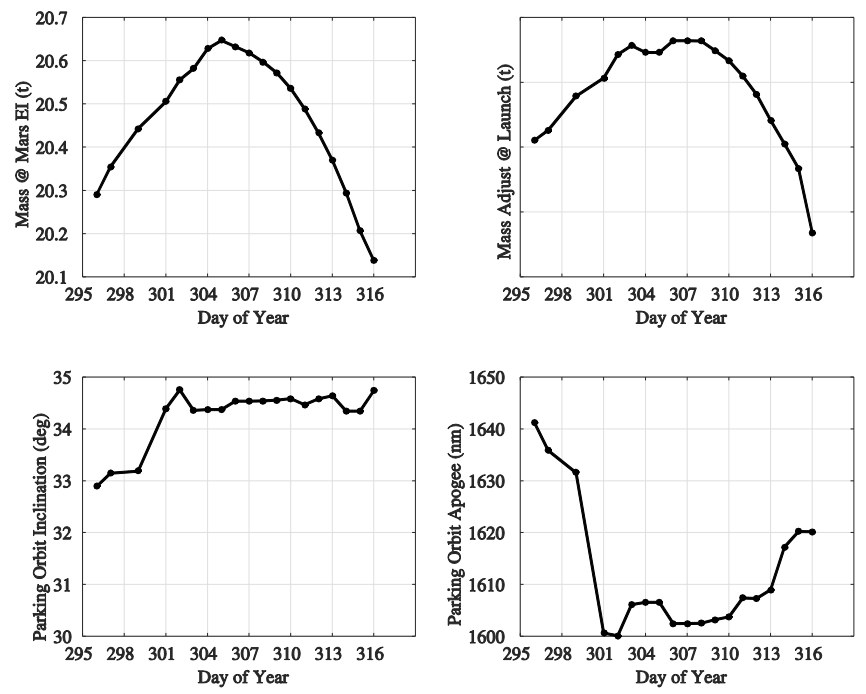

Figure 9. Mars direct entry results

Figure 9 shows launch parameter results as well as the optimized solution. The upper left panel shows the global optimization objective, mass at Mars entry interface. This mass accounts for program payload margin, flight performance reserve, and TMI fuel margin. The value includes the Orion/Stage adapter, and thus is equivalent to the "net payload system mass" shown in Figure 2. A clear maximum of $20.65 \mathrm{t}$ occurs at 1 November 2026, with dropoffs on both sides.

The upper right panel shows the mass adjustment at launch, which is added to the net payload. A maximum is observed at 1 November 2026. Thus, for this day in the launch period, the payload mass must be reduced to achieve maximum mass to Mars entry interface.

The bottom left panel shows parking orbit inclination, which does not exhibit as strong a trend but only varies between $33^{\circ}-35^{\circ}$. Since a perfectly Eastward launch azimuth from Kennedy Space Center yields an inclination of approximately $28.5^{\circ}$, the optimizer is trading SLS launch performance for orbit geometry alignment by launching at azimuths that are not perfectly Eastward.

The bottom right panel shows the variation in parking orbit apogee for the launch period. The location of this apogee is illustrated by the PRM point in Figure 4. Parking orbit apogee ranges from $1600 \mathrm{~nm}$ to approximately $1640 \mathrm{~nm}$. Both parking orbit apogee and inclination here are higher than the optimal values found for EM-1 [1]. This is due to the fact that the payload mass for the Mars direct mission is lower than the lunar mission, allowing the launch vehicle to throw to a higher energy parking orbit. 

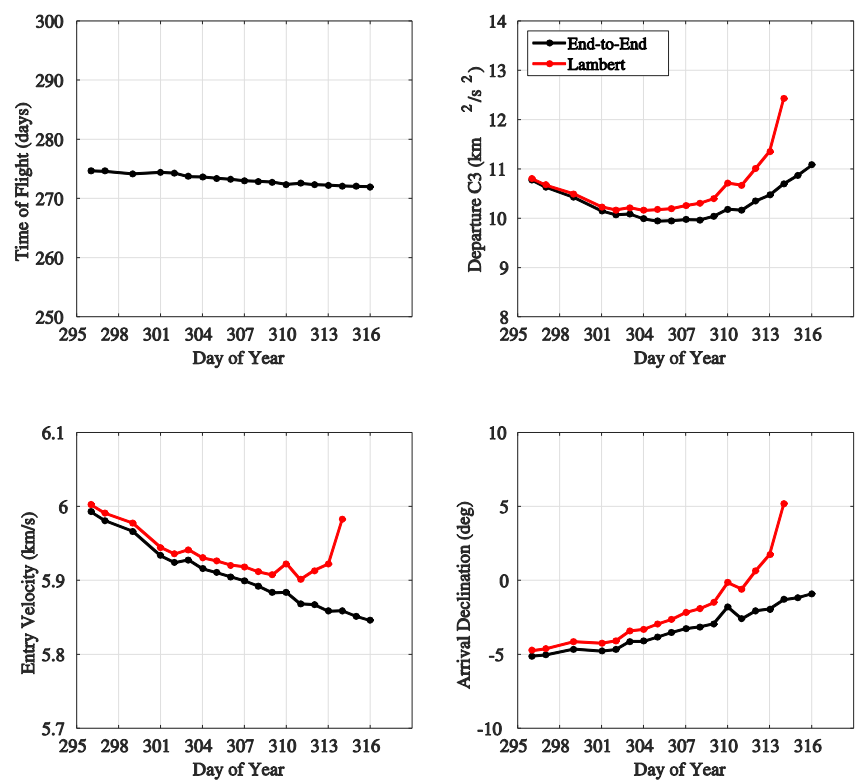

Figure 10. Mars direct entry transfer orbit results

Figure 10 shows transfer orbit results from both the trajectory optimization and Lambert's problem. None of these parameters were constrained in either Copernicus or Isight. The upper left panel shows the time of flight (MECO to Mars entry interface) essentially invariant (272.0 to 274.7 days) across the launch period.

The upper right panel shows the departure characteristic energy with a minimum at 1 November of $9.941 \mathrm{~km}^{2} / \mathrm{s}^{2}$. Entry velocities and arrival declinations, shown in the bottom left and right panels, exhibit essentially linear trends across the launch period.

The solutions from the Lambert problem, shown in red, agree well at the start of the launch period but diverge as the date moves forward. Recall from Figure 5 that the Lambert problem presents a singularity near 12-15 November 2026 . As the launch date approaches this singularity, the Lambert solution will diverge. Thus, while the Lambert solutions provide a good starting point and can quickly highlight favorable ranges of launch dates, the full end-to-end analysis provides more accurate solutions with higher fidelity.

\section{SUMMARY AND CONCLUSIONS}

A robust implementation of an MDAO method that offers the flexibility to quickly adapt to changing mission design requirements was presented. The end-to-end optimization framework is designed to be flexible, robust, and easy to modify, and is itself an extension of the framework used to optimize the EM-1 end-to-end trajectory developed in [1].

A Mars direct entry mission architecture during the 2026 launch opportunity was used as an end-to-end case study. High-fidelity simulation tools were used to model the various phases of the trajectory. The commercial software package Isight was used to combine these tools in an end-to- end optimization framework. Using an SLS Block 1, an optimized maximum net payload to Mars entry interface of $20.65 \mathrm{t}$ occurred on a launch date of 1 November 2026, with a time of flight of 273 days and a corresponding departure C3 of $9.941 \mathrm{~km}^{2} / \mathrm{s}^{2}$. The optimizer in this end-to-end analysis has traded SLS launch performance for improved orbit geometry alignment and achieved a net payload that is comparable to that in the SLS Mission Planner's Guide [2], with higher fidelity and flexibility.

As the SLS program matures and additional launch vehicle simulations are developed, a "library" of simulations will be assembled so that a user may "check out" the simulation for a particular vehicle and quickly implement it in the Isight optimization framework. Ultimately, the flexibility afforded by the MDAO process will permit the user to solve a variety of trajectory design problems posed by mission planners.

\section{ACKNOWLEDGEMENTS}

The authors thank Scott Craig of ERC, Inc./Jacobs ESSSA Group for providing technical guidance and support on SLS vehicle performance and capabilities.

\section{REFERENCES}

[1] Litton, D. K., Lugo, R. A., Qu, M., Craig, A. S., Shidner, J. D., Abebonojo Jr., B. O., Winski, R. G., and Powell, R. W., "Creating an End-to-End Simulation for the Multi-Purpose Crewed Vehicle and Space Launch System," AAS/AIAA Astrodynamics Specialist Conference, Vail, CO, August 2015, AAS 15-641.

[2] "Space Launch System (SLS) Program Mission Planner's Guide (MPG) Executive Overview," Version 1, NASA, SLS-MNL-201, 22 August 2014.

[3] Bate, R. R., Mueller, D. D., and White, J. E., Fundamentals of Astrodynamics, Dover Publications, Inc., New York, 1971.

[4] Striepe, S. A., et al., Program to Optimize Simulated Trajectories (POST2), Vol. 2: Utilization Manual, Ver. 3.0 NESC, NASA Langley Research Center, Hampton, VA, May 2014.

[5] Williams, J., Copernicus Version 4.1 Users Guide, NASA Johnson Space Center, March 2015.

[6] Isight 5.9, Dassault Systèmes Simulia Corp., Providence, RI, Release 5.9-1, 2014.

[7] "The JSON Data Interchange Format," $1^{\text {st }}$ Edition, Ecma International, Standard ECMA-404, October 2013.

[8] Runyan, J., Gerhardt, R. A., and Ruh, R., "Electrical Properties of BN Matrix Composites - Part I: Analysis of the McLachlan Equation in Modeling the Conductivity of BN-B4C and BN-SiC Composites," 
Journal of the American Ceramic Society, Vol. 84, No. 7, 2001, pp. 1490-6.

[9] Gill, P. E., Murray, W., Saunders, M. A., "SNOPT: An SQP Algorithm for Large-Scale Constrained Optimization," SIAM Review, Vol. 47, No. 1, p. 99-131.

[10]Exler, O., Lehmann, T., Schittkowski, K., “A comparative study of SQP-type algorithms for nonlinear and nonconvex mixed-integer optimization," Mathematical Programming Computation, Vol. 4, No. 4, 2012, p.383-412.

\section{BIOGRAPHY}

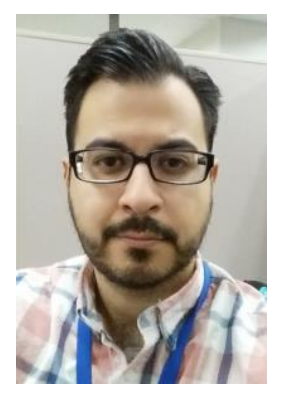

Rafael Lugo holds a B.S., M.S., and Ph.D. in aerospace engineering from North Carolina State University. He is a flight mechanics engineer at Analytical Mechanics Associates, Inc. and is a member of the Atmospheric Flight and Entry Systems Branch at NASA Langley Research Center. While at NASA Langley he has worked on materials testing for inflatable atmospheric decelerators, scale model aeroballistic testing for Orion and MSL, and flight trajectory reconstruction for MSL. He is currently supporting liftoff and trajectory analyses for SLS and atmosphere reconstruction for MAVEN flight operations.

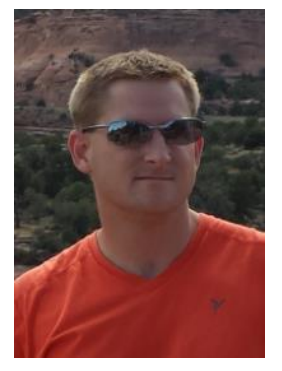

Daniel Litton graduated in 2001 and 2003 with his BS and MS in Aerospace Engineering from North Carolina State University. He first worked at Patuxent River Naval Air Station on F-18 store separation. For the past 11 years he has worked at NASA Langley in the Atmospheric Flight and Entry Systems Branch as a flight dynamics engineer and project manager. He has worked on multiple flight projects including Mars Science Laboratory, Launch Abort System, and the Inflatable Reentry Vehicle Experiment. Currently he is working on the End-to-End simulation, Mars InSight Lander, and leading an acoustic test for the Launch Abort System.

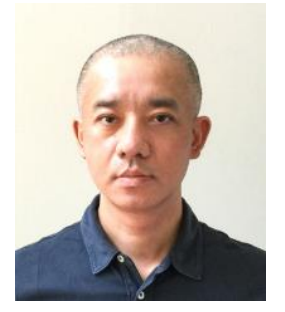

Min $Q u$ received a B.S. from Peking University and an M.S. and Ph.D. in Applied Mathematics from Purdue University. He has been with NASA Langley Research Center for more than 15 years. He is currently supporting the mission analysis and design studies for NASA's Asteroid Redirect Mission (ARM) and Human spaceflight Analysis Team (HAT). Prior to ARM and HAT, he supported various NASA projects and studies including lunar trajectories for Constellation, launch vehicle design and sizing for the Next Generation Launch Technologies (NGLT), tool integration for Advanced Engineering
Environment (AEE), and mass property optimizations for the Orion Abort Flight Test project.

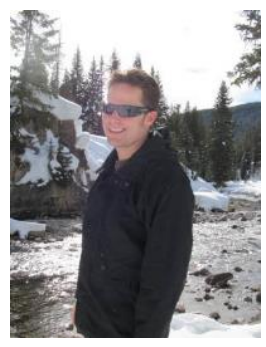

Jeremy Shidner received his B.S. in aerospace engineering from EmbryRiddle Aeronautical University in 2004 and M.S. degree in aerospace engineering from the University of Maryland in 2006. He is currently the director of Flight Dynamics and Control at Analytical Mechanics Associates and is a member of the Atmospheric Flight and Entry Systems Branch at NASA Langley. His current activities include work on the NASA Space Launch System, Commercial Crew Program and POST2 development.

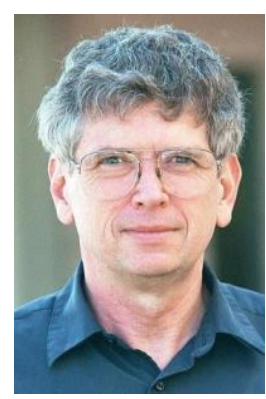

Richard Powell holds a B.S. in Aerospace Engineering from Virginia Tech and a M.S. from George Washington University in Flight Mechanics. His career began in 1970 as a member of the NASA Langley Research Center's Space Systems Research Division that had been formed to provide direct support of the Space Shuttle program. His contributions include being a member of the team that developed a pilot-in-the-loop simulation that was used primarily to test the Shuttle's entry guidance and control systems. He has also worked on many robotic missions and studies, including the Mars Landers (Mars Pathfinder, Mars Phoenix, and Mars Science Laboratory), Earth return vehicles (Stardust), Mars Orbiters (Mars Global Surveyor, and Mars Odyssey, Mars Reconnaissance Orbiter), and European-led missions (Huygens). He retired from NASA in 2007. As a member of Analytical Mechanics Associates, Inc., he is currently working on the Commercial Crew Program, which is designed to allow NASA to purchase transportation to the Space Station, and the Space Launch System designed to be the launch vehicle for NASA to use further solar system exploration including human exploration. 